\title{
Método para la solución de tareas experimentales cualitativas de biología
}

\author{
Dayly García García ${ }^{1}$, Yusimí Guerra Véliz ${ }^{(\mathbb{}}$, Julio Leyva Haza ${ }^{(\mathbb{1}}$ \\ Universidad Central "Marta Abreu" de Las Villas, Cuba
}

Autor de correspondencia: 1daylyg@uclv.cu Recibido: 30 de mayo de 2020 Revisado: 15 julio de 2020 Aprobado: 08 de septiembre de 2020 Publicado: 16 de noviembre de 2020

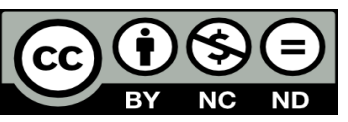

\section{Resumen}

Se aborda la problemática de la baja independencia cognoscitiva de los estudiantes al realizar las prácticas de laboratorio de biología. Para atenuarla, se propone transformar las prácticas de laboratorio tradicionales en tareas experimentales e introducir un método para orientar a los estudiantes en la solución independiente de las tareas experimentales cualitativas de biología. Dicho método fue obtenido por analogía. Para la validación de la propuesta, se realizó un experimento de modelo y una intervención práctica, donde se siguió una secuencia de apertura en una muestra de estudiantes de la Licenciatura en Educación, Biología. Desde el punto de vista estadístico, se aplicó un diseño de bloques completos al azar para comparar la independencia cognoscitiva de los estudiantes en las diferentes etapas del método de solución, y de los estudiantes entre sí. Como resultado del experimento, se evidenció la funcionalidad del método para el diseño de las tareas experimentales correspondientes a las disciplinas biológicas. En la intervención práctica se detectaron regularidades didácticas, que permitieron obtener avances significativos en la independencia cognoscitiva de los estudiantes de la muestra.

Palabras claves: desarrollo de habilidades, experimento, experimento educacional, enseñanza de la biología, formación de docentes. 


\title{
Method to solve qualitative experimental tasks in biology
}

\begin{abstract}
The problem of the low cognitive independence of the students when performing laboratory practices in biology is addressed. To mitigate it, it is proposed to transform traditional laboratory practices into experimental tasks and to introduce a method to guide students in the independent solving of qualitative experimental tasks in biology. The method was obtained by analogy. For the validation of the proposal, a model experiment and a practical intervention were carried out following an opening sequence in a sample of students from the Bachelor of Education, Biology career. From the statistical point of view, a randomized complete block design was applied to compare the cognitive independence of the students in the different stages of the solving method, and of the students among themselves. As a result of the model experiment, the functionality of the method for the design of the experimental tasks corresponding to the biological disciplines was evidenced. In the practical intervention, didactic regularities were detected, which allowed obtaining significant advances in the cognitive independence of the students in the sample.
\end{abstract}

Keywords: skill development, experiment, educational experiment, biology teaching, teacher training

\section{Método para a solução de tarefas experimentais qualitativas em biologia}

\section{Resumo}

O problema da baixa independência cognitiva dos estudantes na realização de práticas laboratoriais de biologia é abordado. Para atenuá-lo, propõe-se transformar as práticas laboratoriais tradicionais em tarefas experimentais e introduzir um método para orientar os estudantes na solução independente de tarefas experimentais qualitativas em biologia. Este método foi obtido por analogia. Para a validação da proposta, foi realizado um experimento modelo e uma intervenção prática, onde foi seguida uma sequência de abertura numa amostra de estudantes da Licenciatura em Educação, Biologia. Do ponto de vista estatístico, foi aplicado um desenho de blocos completos aleatórios para comparar a independência cognitiva dos estudantes nas diferentes fases do método de solução, e dos estudantes entre si. Como resultado do experimento, foi evidenciada a funcionalidade do método para o desenho das tarefas experimentais correspondentes às disciplinas biológicas. $\mathrm{Na}$ intervenção prática, foram detectadas regularidades didáticas, que permitiram obter avanços significativos na independência cognitiva dos estudantes da amostra.

Palavras-chave: desenvolvimento de habilidades, experimento, experimento educacional, ensino de biologia, formação de professores. 
Benoit et al. (2019) afirman que dos de los obstáculos que afectan la educación universitaria actual son el alto nivel de deserción y el bajo rendimiento académico de los estudiantes. Dicen que ambas son "dificultades que pueden experimentar los estudiantes a lo largo de su proceso de formación” (p. 92).

Las causas de la deserción y del bajo rendimiento académico pueden ser múltiples, pero es indiscutible que en los procesos de enseñanza aprendizaje universitarios "tratar de captar la atención de los estudiantes, utilizando estrategias para generar motivación, curiosidad e interés, debe formar parte de la tarea del docente" (Álvarez \& Valls, 2019, p. 74).

Algunas estrategias, cuando se trata de la formación en ciencias naturales, pueden ser el trabajo en el laboratorio y la experimentación como parte de las prácticas científicas. "No se trata tan solo de enseñar conocimientos científicos, sino también de enseñar los métodos utilizados por la ciencia para obtenerlos" (Ferrés-Gurt, 2017, p. 411). "Las prácticas de laboratorio concebidas como estrategias didácticas deben permitirles a los estudiantes comprender la forma en que se construye el conocimiento en una comunidad científica" (Espinosa-Ríos et al., 2016, p. 269).

"El trabajo de laboratorio se considera un entorno adecuado para el aprendizaje a través de la indagación, sin embargo, las prácticas habituales de laboratorio no siempre permiten alcanzar estos objetivos" (Hodson citado por Crujeiras \& Jiménez, 2015, p. 63). En las prácticas de laboratorio habituales ha prevalecido el carácter reproductivo en que los estudiantes siguen las indicaciones de un guion que los conduce a realizar operaciones sin previo análisis, fenómeno didáctico conocido como tendencia a la ejecución (Labarrere, 1996). Los estudiantes se limitan a realizar una rutina sin comprender, en la mayoría de los casos, lo que han hecho. Esta situación es documentada por varios autores, entre ellos González et al. (2009), Carp et al. (2012) y Fernández (2013).

Según Landau et al. (1997), esta metodología, estructurada y rígida, no da lugar a la formación de nuevos conocimientos en los estudiantes y restringe su independencia cognoscitiva.

Estas razones evidencian la necesidad de un cambio que acerque más al estudiante al trabajo del científico. "El enfoque de aprendizaje a través de las prácticas científicas implica, entre otras cuestiones, que el alumnado utilice y desarrolle determinadas destrezas, no solo procedimentales, sino también de razonamiento" (Crujeiras \& Cambeiro, 2018, p. 1201-2)

Para Van der Valk y De Jong (citados por Siso et al., 2009), el estudiante debería participar en el diseño de actividades de laboratorio, posición que es complementada por Domenech (2013) al plantear la necesidad de que, "a partir del interés y el estímulo, el estudiante reflexione, se apropie de estrategias y secuencias de apertura experimental en el laboratorio y construya un modelo coherente, sólido que le ayude a entender el mundo" (pp. 250-251).

En este trabajo se propone una transformación de las prácticas de laboratorio reproductivas para presentarlas como tareas experimentales. Se propone un método de solución, que, de manera heurística, oriente al estudiante en su solución. Tal método lleva a entender "las actividades experimentales como contenido" (Peres \& Marques, 2013, p. 68), puesto que "son relevantes para el aprendizaje de contenidos conceptuales, procedimentales y actitudinales" (p. 72), lo cual las convierte en un recurso cognitivo para aplicar los conocimientos a situaciones reales. 
El objetivo de este trabajo es presentar una estructura de carácter heurístico para el método de solución de tareas experimentales cualitativas de biología que, como contenido, contribuya a la independencia cognoscitiva de estudiantes de profesorado de esta especialidad, puesto que "mejorar la actuación del profesorado en el aula es la principal finalidad de toda actividad de desarrollo profesional" (Gil, 2017, p. 177).

\section{El método de solución de tareas experimentales cualitativas de biología como heurístico}

En la estructura del proceso enseñanza-aprendizaje, el método aparece dos veces: como uno de sus componentes y como parte del contenido, que es, a su vez, otro de sus componentes.

En el primer caso, "el método es el orden y se basa en la consecutividad de las acciones que ejecuta cada estudiante para aprender y cada profesor para enseñar" (Muschietti-Piana et al., 2017, p. 33).

En el segundo caso, el método es parte del contenido y se refiere a los métodos de la rama del saber que se estudia y son los que determinan las habilidades a desarrollar. Es decir, tienen la función de "ayudar a los estudiantes a hacer ciencia" (Barolli et al., 2010, p. 96).

Según las exigencias del modelo del profesional de la carrera Licenciatura en Educación, Biología, Plan E (Ministerio de Educación Superior, 2016), el estudiante debe "resolver tareas nuevas que surgen cotidianamente, desarrollar la habilidad de trabajar independientemente en la adquisición de conocimientos e interactuar con el desarrollo de la ciencia y la técnica, lo cual es un imperativo de la sociedad actual" (p. 7).

La solución de tareas experimentales cualitativas de biología exige deducciones lógicas del estudiante, a partir de su sistema de conocimientos prácticos y teóricos, al buscar la vía más adecuada para solucionarlas. "Las ciencias biológicas se caracterizan por utilizar, sobre la base de un marco teórico, la observación y la experimentación con los organismos para la construcción de conocimiento" (Grilli-Silva, 2018, p. 1104-1).

Por ello, contar con la estructura del método de solución de las tareas experimentales cualitativas como parte del contenido es un heurístico que puede ser usado para orientarse en la solución de dichas tareas, a la vez que propicia la independencia cognoscitiva.

En la bibliografía consultada se han encontrado varias propuestas que pueden ser consideradas estructuras del método de solución de tareas experimentales. Se tomaron como referencia las formuladas por Kiruschkin et al. (1981), Hodson (1994), Caamaño (2012), Domenech (2013) y González y Crujeiras (2016), en las que se advierten las siguientes regularidades:

1. Transferir, más o menos directamente, el método de investigación científica a la enseñanza.

2. Las etapas que componen la estructura del método de solución de tareas

experimentales son obtenidas por vía empírica a partir de la práctica pedagógica.

La primera regularidad tiene el inconveniente de no considerar las particularidades del proceso de enseñanza aprendizaje que lo distinguen del proceso de investigación científica. "Las acciones experimentales, que corresponden al máximo nivel, según Miller, cuando son 
autónomas (al ser realizadas por profesionales), al ser enseñadas en la escuela, requieren otras competencias específicas de la enseñanza” (Via \& Izquierdo, 2016, p. 86).

La segunda lleva a que no exista consenso en el número de etapas del método o en su contenido, al no reflejar la esencia del hecho didáctico al cual dicho método se refiere.

En consecuencia, para establecer la estructura del método de solución de tareas experimentales cualitativas de biología, se tuvo en cuenta, por un lado, considerar las particularidades del proceso de solución de este tipo de tareas en el proceso de enseñanzaaprendizaje y, por otro, la conjunción de su determinación teórica y empírica.

Estos presupuestos son equivalentes a los propuestos por Leyva (2002) para las tareas experimentales cuantitativas de física, por lo que la estructura del método solución de tareas experimentales cualitativas de biología se obtuvo al tomar como referencia el resultado obtenido por este autor y al considerar las especificidades de las tareas experimentales cualitativas de biología.

La estructura del método de solución de tareas experimentales cualitativas de biología quedó conformada por las siguientes etapas: comprensión de la tarea, confección del plan de la solución, ejecución del plan de la solución, diseño del experimento, ejecución del experimento $y$ procesamiento de los datos, control valorativo del curso de la solución y de los resultados, contrastación teórica de los resultados y proposición de la nueva tarea (García, 2019).

Una particularidad de las tareas experimentales cualitativas de biología se concreta en que en cada tarea es necesario dilucidar si se trata de un fenómeno biológico o de una estructura biológica a observar - pueden ser ambas-.

La etapa confección del plan de la solución para las tareas experimentales cualitativas puede pasar inadvertida, incluso para quien resuelve la tarea, por cuanto son acciones que, en la mayoría de los casos, se suceden de manera mental, sin apoyo externo — materializado-. Es decir, no resulta imprescindible, por ejemplo, escribir o dibujar algo. Por tal razón, es aconsejable que el docente oriente la actividad de los estudiantes, mediante preguntas, para lograr que reconozcan de forma explícita la utilidad de esta etapa y transiten por ella.

Por tal razón, en esta etapa, de carácter teórico, se ponen en juego los conocimientos biológicos, relacionados con la consigna y con el fenómeno o la estructura biológica de que trata la tarea experimental. Esta es la base sobre la cual se ideará, posteriormente, la solución experimental de la tarea.

La solución experimental comienza con la etapa de diseño del experimento, para lo cual se propuso un cuadro que, como recurso didáctico, orienta al estudiante a diseñar el experimento (García et al., 2017).

En el resto de las etapas, evidentemente, se opera con contenidos biológicos. Sin embargo, el modo de proceder resulta análogo al declarado por Leyva (2002) y confirmado en su investigación por García (2019).

La determinación de los procedimientos mediante los cuales se realiza cada etapa se sustentó en diversas fuentes bibliográficas (Campistrous \& Rizo, 2002; Galagovsky \& Adúriz-Bravo, 2001; Holyoak citado por Gómez et al., 2013; Labarrere, 1996; Machado, 2004; Rivero, 2003), cuya síntesis y adecuación llevó a los resultados de la tabla 1. 


\section{Tabla 1}

Procedimientos mediante los que se realizan las etapas del método de solución de tareas experimentales cualitativas de biología

\begin{tabular}{l} 
Comprensión de la tarea \\
\hline Lectura global \\
Lectura analítica \\
Análisis semántico \\
Modelación de la situación de la tarea \\
Organizador de datos \\
Reformulación de la tarea \\
\hline Confección del plan de la solución \\
\hline Establecimiento del área de búsqueda \\
Empleo de subtareas auxiliares \\
\hline \multicolumn{1}{c}{ Ejecución del plan de la solución } \\
\hline Diseño del experimento \\
\hline Contrastación teórica de los resultados \\
\hline Cuadro de diseño del experimento \\
\hline Ejecución del experimento y procesamiento de los datos \\
\hline Comparolisis de la significación de los resultados \\
\hline Enunciadorativo del curso de la solución y de los resultados \\
\hline
\end{tabular}

En la tabla 1 se puede apreciar que las etapas correspondientes a la ejecución no disponen de procedimientos, por cuanto ellas se realizan mediante el cumplimiento del plan concebido en la etapa anterior.

\section{Metodología}

Para la validación del método de solución de tareas experimentales cualitativas de biología, se siguió un paradigma mixto. Esta se realizó en dos partes: primero, se aplicó un experimento de modelo con el propósito de probar la pertinencia del método en el diseño de dos asignaturas; y después, se intervino en la práctica para probar la funcionalidad didáctica de dicho método.

Guerra (2008), al referirse a los experimentos de modelo en didáctica, plantea que "sin llegar a ejecutar el proceso docente educativo en sí, un experimento de modelo puede ser el diseño de la asignatura" (p. 106). El método de solución de tareas experimentales cualitativas de biología puede ser analizado como un modelo generalizado de la ejecución procedimental al resolver este tipo de tareas. 
El experimento de modelo estuvo constituido por:

1. Elaboración de tareas experimentales cualitativas de biología para comprobar que podía enunciarse un número suficientemente grande de estas, como para que tuvieran representatividad en todas las disciplinas biológicas del currículo de formación de profesores de esta especialidad.

2. Solución por el docente de las tareas experimentales elaboradas para comprobar la validez del método de solución de tareas experimentales cualitativas de biología, de los procedimientos que lo integran y de las especificidades del método.

3. Diseño del proceso de enseñanza-aprendizaje de las asignaturas seleccionadas para realizar la intervención en la práctica del método de solución de tareas experimentales cualitativas de biología.

Para la intervención en la práctica — dirigida a probar la funcionalidad didáctica del método-, se aplicó un diseño experimental de series cronológicas múltiples con tres mediciones de la variable dependiente. Como variable independiente se consideró el método de solución de tareas experimentales cualitativas de biología; y como variable dependiente, la independencia cognoscitiva de los estudiantes en la solución de tareas experimentales cualitativas de biología.

Para analizar el comportamiento de la variable dependiente, se trabajó con un diseño de bloques completos al azar, conformado por tres ensayos, a cada uno de los cuales corresponde una medición de la variable dependiente. Antes de la primera medición, se realizó la introducción de los elementos estructurales del método de solución de tareas experimentales cualitativas de biología. Entre dos mediciones consecutivas se introdujeron correcciones didácticas a la variable independiente en correspondencia con los resultados que se iban obteniendo.

A cada medición se le llamó ensayo $\left[\mathrm{E}_{i}\right]$; al periodo de intervención anterior a la primera medición se le denominó fase de dominio inicial [FDI]; al periodo de intervención entre las dos primeras mediciones se le llamó fase de dominio formal [FDF]; y al otro periodo transcurrido entre las dos mediciones restantes se le llamó fase de consolidación [FC] (figura 1). Estos nombres están relacionados con los resultados obtenidos en cada periodo.

\section{Figura 1}

Representación gráfica de la secuencia de intervención en la práctica

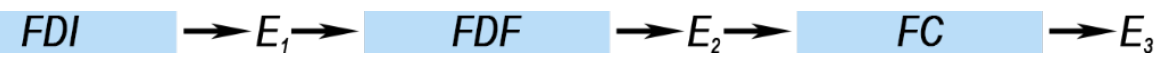

Este modo de concebir la intervención permitió considerar que "el apoyo del experto no es solo para ayudar al aprendiz en la resolución de tareas, sino también para mejorar sus desempeños en tareas futuras" (Crujeiras \& Jiménez, 2018, p. 23), puesto que "la formación y el desarrollo de las habilidades experimentales deben transitar por una serie de etapas que permitan su iniciación, formación y desarrollo" (Hernández-Junco et al., 2018, p. 320).

Al considerar la solución de tareas experimentales cualitativas de biología como una habilidad extendida (Leyva \& Guerra, 2005), se diseñaron para el estudio 21 tareas experimentales de dos asignaturas consecutivas del currículo - primer y segundo semestres de la carrera-. La primera, El desarrollo de habilidades para el trabajo con el material 
biológico, fue especialmente diseñada como currículo propio para enseñar a los estudiantes a resolver tareas experimentales de biología con el método propuesto. La segunda, Biología celular y molecular $I$, pertenece al currículo básico. Para la primera asignatura se diseñaron 12 tareas y 9 para la segunda.

\section{Figura 2}

Secuencia de apertura del método de solución de tareas experimentales cualitativas de biología.

$\begin{array}{llllllllllllllllllllll}\text { Tareas } & 1 & 2 & 3 & 4 & 5 & 6 & 7 & 8 & 9 & 10 & 11 & 12 & 1 & 2 & 3 & 4 & 5 & 6 & 7 & 8 & 9\end{array}$

\section{Comprensión de la tarea}
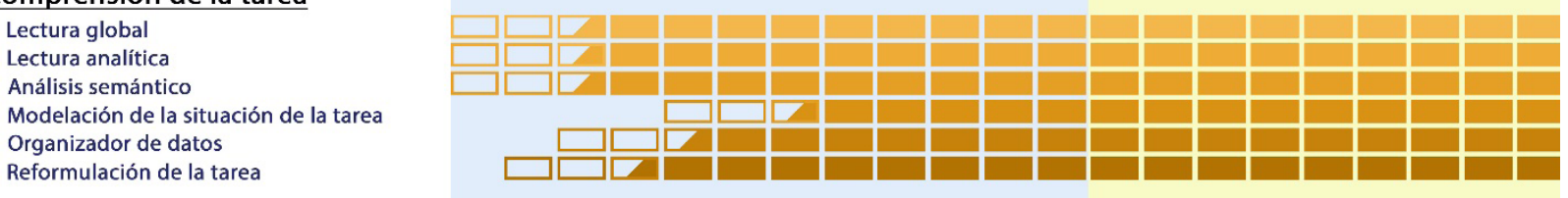

Confección del plan de la solución

Establecimiento del área de búsqueda

Empleo de subtareas auxiliares

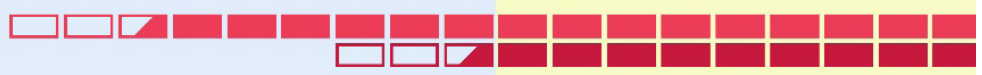

\section{Ejecución del plan de la solución}

Ejecución

Diseño del experimento

Cuadro de diseño del experimento

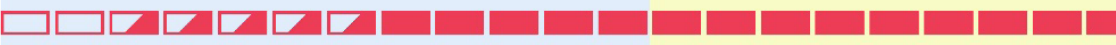

Ejecución del experimento y procesamiento de los datos Ejecución

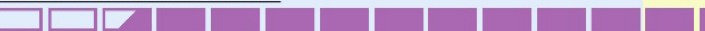

\section{Control valorativo del curso de la solución y de los resultados}

Ajuste al pronóstice

Comparación con vías alternativas

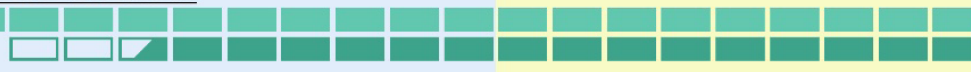

\section{Contrastación teórica de los resultados}

Análisis de la significación de los resultados Valoración del carácter de verdad

\section{Proposición de la nueva tarea}

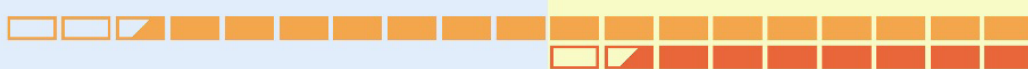

$$
\text { Leyenda: } \square \text { muestra } \square \text { apertura } \square \text { consolidación } \quad \text { incidental }
$$

Como método didáctico para la introducción paulatina de la estructura del método de solución de tareas experimentales cualitativas de biología, se utilizó una secuencia de apertura (Domenech, 2013) (figura 2), a fin de alcanzar gradualmente la independencia cognoscitiva de los estudiantes.

La secuencia de apertura, de acuerdo con la independencia cognoscitiva del estudiante durante la solución de la tarea experimental cualitativa de biología, se ha concebido en tres eslabones: muestra, apertura y consolidación. En la muestra predomina la actividad del profesor que aplica determinado procedimiento para que sirva de ejemplo al estudiante. En la apertura predomina el trabajo parcialmente independiente del estudiante con niveles de ayuda ofrecidos por el profesor. En la consolidación predomina el trabajo independiente del estudiante puesto que la habilidad adquiere solidez y sistematicidad (García, 2019, pp. 103-104).

En la secuencia de apertura aparece además el eslabón incidental, para identificar la 
implicación espontánea de los alumnos en el enunciado de la nueva tarea, aun cuando el profesor no había introducido formalmente este procedimiento, ni la etapa que le da lugar.

\section{Resultados y discusión}

\section{Experimento de modelo}

Como resultado del experimento de modelo, se elaboraron y se resolvieron, aplicando el método de solución propuesto, 167 tareas experimentales cualitativas de biología distribuidas del siguiente modo: biología molecular y celular - 37-, microbiología - 35-, botánica $-43-$, zoología -23 - anatomía y fisiología $-28-$ y ecología $-21-$. Se diseñaron las dos asignaturas consideradas en las que se incluyeron las 21 tareas para llevar a cabo la secuencia de apertura (figura 2).

Discusión de los resultados del experimento de modelo. Haber podido resolver las tareas experimentales elaboradas es una prueba de la validez del método de solución propuesto y de su generalidad. Con ello se confirmó, además, la validez de la estructura del método de solución de tareas experimentales cualitativas de biología, aunque fue necesario agregar al cuadro de diseño del experimento el factor forma de procesamiento de los datos e intercambiar de lugar los factores fenómeno a reproducir y estructura a observar, que hasta el segundo paso del experimento de modelo estaban a la inversa (García et al., 2017). Tal cambio respondió al hecho de que un estudio experimental puede realizarse sobre una estructura biológica sin considerar un fenómeno, pero todo fenómeno biológico se da asociado a una estructura, de modo que resulta más fácil identificar la estructura a observar que el fenómeno a reproducir.

\section{Intervención en la práctica}

Contexto y participantes. Se analiza el desempeño de todos los estudiantes del grupo de primer año de Licenciatura en Educación, Biología del curso 2016-2017 - 10 estudiantesque constituyen la muestra.

Su aprovechamiento académico es medio. Tienen pocos hábitos de estudio, aunque todos aprobaron los exámenes de ingreso a la educación superior y nunca se han enfrentado a la solución de tareas experimentales. Solo dos estudiantes graduados del curso de nivel medio de profesores de biología-química han realizado prácticas de laboratorio, pero según el estilo tradicional. La información anterior se obtuvo de la entrega pedagógica, de la revisión de expediente acumulativo del escolar y de una entrevista abierta realizada a los estudiantes.

Diseño estadístico de la intervención práctica: secuencia de apertura. Las variables independientes y dependientes se relacionaron en la siguiente hipótesis de trabajo: si se dispone de la estructura del método de solución de tareas experimentales cualitativas de biología como contenido, se obtendrán avances progresivos en la independencia cognoscitiva de los estudiantes para la solución de tareas experimentales cualitativas de biología.

En el diseño en bloques completos al azar - que se llevaría a cabo en cada ensayo-, se consideran las 8 etapas del método y los estudiantes de la muestra que determinan 10 bloques. Se mide la independencia cognoscitiva de cada estudiante al ejecutar cada etapa. Los resultados de la medición se recogieron en una tabla de 8 por 10. Cada celda de la 
tabla contiene la calificación de la independencia cognoscitiva del estudiante $j$ en la etapa $i$ del método. Para cada ensayo se obtuvo una tabla cuyos datos se tomaron para realizar las pruebas de hipótesis. Las calificaciones se otorgaron según la escala de $2-$ mal-, $3-$ regular-, 4 - bien- y 5 - excelente-.

Acciones para la intervención práctica. Comparar las etapas del método entre sí con respecto al logro de la independencia cognoscitiva de los estudiantes al solucionar una tarea experimental.

1. Establecer grupos homogéneos para las etapas del método, determinados por las diferencias encontradas en la acción anterior.

2. Comparar los estudiantes entre sí.

Los resultados obtenidos en la primera y en la segunda acción permiten tomar medidas didácticas para el trabajo con las etapas del método en las que se alcancen resultados bajos. La tercera acción permite trazar una estrategia de atención a las diferencias individuales de los estudiantes.

Las parejas de hipótesis a contrastar son tres, una para cada acción.

\section{Para la primera acción.}

H0: No existen diferencias entre las etapas del método, en cuanto a la independencia cognoscitiva de los estudiantes.

H1: Existen diferencias entre las etapas del método, o al menos existe diferencia en una de ellas, en cuanto a la independencia cognoscitiva de los estudiantes.

Para probar estas hipótesis se usó la prueba de los efectos intersujetos para factores fijos, tomando como factor fijo cada una de las ocho etapas del método de solución.

\section{Para la segunda acción.}

H0: Las etapas del método de solución pertenecen al mismo grupo homogéneo.

H1: Las etapas del método de solución pertenecen a diferentes grupos homogéneos.

Para probar estas hipótesis se usa la prueba de Duncan, que permite formar los grupos homogéneos para las etapas del método de solución de tareas experimentales cualitativas de biología.

\section{Para la tercera acción.}

H0: No existen diferencias entre los estudiantes en cuanto a la independencia cognoscitiva al aplicar las etapas del método de solución.

H1: Existen diferencias entre los estudiantes, o al menos existe diferencia en uno de ellos, en cuanto a la independencia cognoscitiva al aplicar las etapas del método.

Para probar estas hipótesis se usó la prueba de los efectos intersujetos para factores fijos, con el factor fijo estudiante. El nivel de significación en todas las pruebas fue $\alpha=0,05$.

Resultados y discusión de la intervención práctica. Como la intervención práctica se realizó siguiendo un proceso de series cronológicas múltiples no establecidas previamente, cada resultado posterior está condicionado por el anterior. Por ello, en este apartado, una 
vez que se presenta un resultado, inmediatamente se hace su discusión, de modo que se pueda apreciar cómo el resultado subsiguiente es concebido.

En la fase de dominio inicial, se presentaron dificultades en los procedimientos de las etapas comprensión de la tarea y confección del plan de la solución, pues se observó una marcada tendencia a la ejecución, al tratar de hacer el cuadro de diseño del experimento sin realizar la solución teórica. Para resolver esta dificultad, se ofrecieron niveles de ayuda. A la altura de la tarea 5, ya muchos estudiantes mostraban alguna independencia en los procedimientos, que se consolidaron hasta la tarea 9.

El primer ensayo se realizó en la tarea 1 de Biología molecular y celular I — tarea 9 de la intervención práctica-. Los resultados son los siguientes:

- La prueba de efectos intersujetos para el factor fijo etapas del método de solución arrojó una significación de $0,000<0,05$, lo que indica diferencias significativas en la independencia cognoscitiva en la aplicación de las atapas método.

- La prueba de Duncan arroja dos grupos homogéneos para las etapas del método (tabla 2). Además, aunque las medias para el segundo grupo están por encima de tres puntos - aprobados-, sus valores son muy bajos.

Tabla 2

Prueba de Duncan para las etapas del método de solución de tareas experimentales cualitativas de Biología. Primer ensayo.

\begin{tabular}{llcc}
\multicolumn{2}{c}{$\begin{array}{c}\text { Variable dependiente: independencia cognoscitiva en la aplicación del método de solución } \\
\text { de tareas experimentales cualitativas de biología }\end{array}$} \\
\hline \multicolumn{1}{c}{ Etapas método } & N & \multicolumn{2}{c}{ Grupos homogéneos } \\
\cline { 3 - 4 } & & Grupo 1 & Grupo 2 \\
\hline Contrastación teórica de los resultados & 10 & 2,20 & \\
Proposición de la nueva tarea & 10 & 2,30 & \\
Comprensión de la tarea & 10 & & 2,90 \\
Control valorativo & 10 & & 2,90 \\
Confección del plan de la solución & 10 & & 3,00 \\
Ejecución del experimento y procesamiento de los datos & 10 & & 3,20 \\
Ejecución del plan de la solución & 10 & & 3,20 \\
Diseño del experimento & 10 & & 3,30 \\
Sig. & &, 649 &, 114 \\
\hline
\end{tabular}

Los valores alcanzados en la independencia cognoscitiva en el primer ensayo indican un nivel bajo para cada una de las etapas. Estos resultados sugieren que el número de tareas resueltas aún no es suficiente, por lo que, para alcanzar niveles superiores, los estudiantes deben resolver un número mayor de tareas experimentales. Tales resultados apuntan, además, hacia una primera acción didáctica encaminada a intensificar el trabajo con todas las etapas del método.

La existencia de dos grupos homogéneos en las etapas del método indica que la independencia cognoscitiva no se logra por igual en todas las etapas. Las etapas del primer 
grupo resultaron más difíciles para los estudiantes. Suponemos que ello se debe a que dichas etapas son las últimas que han quedado abiertas según la secuencia de apertura. A partir de estos resultados se sugiere una segunda acción didáctica encaminada a enfatizar el trabajo con las etapas del primer grupo.

- La prueba de efectos intersujetos para el factor fijo estudiante devolvió el valor de significación de $0,000<0,05$, que indica diferencias significativas entre ellos.

En el caso de las medias por estudiantes, solo tres están por encima de tres puntos: el estudiante 5, el 7 y el 9. El resto está por debajo (tabla 3, primer ensayo). Esto sugirió una tercera acción didáctica encaminada a trabajar de manera diferenciada con los estudiantes cuyas calificaciones están por debajo de 3 puntos.

\section{Tabla 3}

Promedio de las calificaciones por estudiante en cada ensayo

\begin{tabular}{cccc}
\hline Estudiante & Primer ensayo & Segundo ensayo & Tercer ensayo \\
\hline 1 & 2,75 & 3,38 & 2,75 \\
2 & 2,50 & 2,25 & 2,50 \\
3 & 2,75 & 3,88 & 3,38 \\
4 & 2,75 & 3,25 & 3,38 \\
5 & 3,63 & 4,25 & 4,63 \\
6 & 2,88 & 3,00 & 3,38 \\
7 & 3,75 & 4,38 & 4,63 \\
8 & 2,38 & 3,00 & 3,00 \\
9 & 3,13 & 3,75 & 4,38 \\
10 & 2,25 & 3,00 & 2,75 \\
\hline
\end{tabular}

De modo general, los resultados de este ensayo, tanto para el factor fijo etapas del método, como para el factor fijo estudiantes, se consideran bajos. Por esto, se consideró seguir con la secuencia de apertura. Otro aspecto que pudo haber influido en los resultados es el hecho de que la tarea experimental evaluada es la primera que se resuelve en Biología molecular y celular I, de modo que, desde la última tarea resuelta en el semestre anterior, los estudiantes han pasado un periodo largo sin enfrentarse a la solución de este tipo de tareas.

Las acciones didácticas se pusieron en práctica en la fase de dominio formal. Mediante la observación participante, se detectó que los estudiantes seguían manifestando tendencia a la ejecución, expresada en el paso directo a la obtención de la solución teórica, sin trazarse un plan previo.

La complejidad de la solución teórica, que en este tipo de tareas requiere de indagaciones bibliográficas e incluso en internet, se comportó como una barrera para avanzar en dicha solución, por ser la base para el diseño del experimento. Ello condujo a orientar las tareas experimentales con varias clases de antelación y a realizar consultas y controles intermedios para aclarar dudas y valorar los avances en la solución teórica. Esta medida exigió un trabajo adicional para el profesor, puesto que, en la mayoría de los casos, las consultas deben ser individualizadas para tener un control del aprendizaje de cada estudiante según el diagnóstico. 
Se aplicó además una entrevista a los estudiantes en la que expresaron que el procedimiento organizador de datos les resultó de mucha ayuda, puesto que les permitía desplegar estrategias de aprendizaje propias para la solución teórica de la tarea experimental. Señalaron, además, como un aspecto interesante, la posibilidad de que cada uno podía conformar una solución diferente y podían compararla con otros.

El segundo ensayo se realizó en la tarea 6 de Biología molecular y celular I. Esta tarea se orientó con antelación y se ofrecieron consultas previas e individualizadas a los estudiantes.

El objetivo e hipótesis del segundo ensayo son similares a los del primero. Los resultados son los siguientes:

- La prueba de efectos intersujetos para el factor fijo etapas del método arrojó una significación de $0,07>0,05$, que indica que no existen diferencias entre dichas etapas. Aunque la cercanía entre 0,07 y 0,05 sugiere una significatividad débil.

- Dada la debilidad de significatividad, la prueba de Duncan arroja dos grupos de etapas (tabla 4). Sin embargo, la diferencia entre dichos grupos solo se da para la contrastación teórica de los resultados, que es la más afectada con una media de 3,10, posiblemente por ser la última en mostrarse y quedar abierta a los estudiantes en la tarea 5.

\section{Tabla 4}

Prueba de Duncan para las etapas del método de solución de tareas experimentales cualitativas de biología. Segundo ensayo.

\begin{tabular}{lccc}
\hline \multicolumn{4}{c}{$\begin{array}{c}\text { Variable dependiente: independencia cognoscitiva en la aplicación del método de solución de } \\
\text { tareas experimentales cualitativas de biología }\end{array}$} \\
\hline \multicolumn{1}{c}{ Etapas método } & $\mathbf{N}$ & Grupos homogéneos \\
\cline { 3 - 5 } & 10 & 3,10 & Grupo 1 \\
\hline Contrastación teórica de los resultados & 10 & 3,20 & 3,20 \\
Control valorativo & 10 & 3,40 & 3,40 \\
Comprensión de la tarea & 10 & 3,40 & 3,40 \\
Proposición de la nueva tarea & 10 & 3,50 & 3,50 \\
Ejecución del plan de la solución & 10 & 3,50 & 3,50 \\
Ejecución del experimento y procesamiento de los datos & 10 & & 3,60 \\
Confección del plan de la solución & 10 & & 3,60 \\
Diseño del experimento & &, 056 &, 059 \\
Sig. & & & \\
\hline
\end{tabular}

Las etapas confección del plan de la solución y diseño del experimento son las de mejores resultados con una media de 3,60, quizás por todo el énfasis que se puso en ellas después de los resultados del primer ensayo. El resto de las etapas del método de solución pertenecen a los dos grupos y ello da una idea de la tendencia a formarse un único grupo en la independencia cognoscitiva respecto a las etapas del método.

Como todas las medias para la independencia cognoscitiva en las etapas del método de solución estaban por debajo de 3,60, se decidió trazar una primera acción didáctica, para 
la siguiente etapa, consistente en seguir trabajando en pos de mejorar los resultados. Así mismo, el hecho de que el rango de los puntajes de las etapas fuera pequeño $(3,10-3,60)$ sugirió una segunda acción didáctica encaminada a trabajar con todas ellas, reforzando un poco más la contrastación teórica de los resultados que fue la más afectada $(3,10)$.

- La prueba de los efectos intersujetos para el factor fijo estudiantes devolvió el valor de significación de Esto evidencia que existen diferencias entre los estudiantes, aunque se observa que todos $0,000<0,05$. mejoraron sus calificaciones respecto al primer ensayo y, además, todas las medias están por encima de 3,00 (tabla 3, segundo ensayo).

El hecho de que solo dos estudiantes alcancen medias mayores que 4,00 sugiere una tercera acción didáctica dirigida a intensificar más el trabajo diferenciado con los estudiantes que alcanzan las menores calificaciones.

Las acciones didácticas trazadas a partir de los resultados del segundo ensayo se pusieron en práctica en la fase de consolidación. Durante la realización de todas las tareas correspondientes a esta fase, se observaron avances en los estudiantes, aunque el estudiante 10 comenzó a tener ausencias y llegadas tarde a clases que se reflejaron en resultados bajos en su evaluación frecuente.

En las últimas tareas los resultados de las evaluaciones intermedias disminuyeron en algunos casos. Según entrevistas abiertas realizadas a los estudiantes, ellos manifestaron que tenían que estudiar para el resto de las asignaturas del currículo y que se acercaban, además, los exámenes finales.

El tercer ensayo se realizó en la tarea 9 de Biología molecular y celular I. Esta tarea se orientó con antelación y se ofrecieron consultas individualizadas a los estudiantes.

El objetivo e hipótesis del tercer ensayo son similares a los del primero y segundo. Los resultados de este ensayo son los siguientes:

- La prueba de efectos intersujetos para el factor fijo etapas del método de solución arrojó una significación de $0,255>0,05$, que indica que no existen diferencias significativas en la independencia cognoscitiva respecto a las etapas del método de solución.

- En la prueba de Duncan para determinación de grupos homogéneos para las etapas del método de solución, se observa la formación de un único grupo de etapas, lo que se corresponde con el alto grado de significatividad $-0,255$ - de la prueba de efectos intersujetos (tabla 5).

Dado que todas las medias para las etapas del método de solución están entre 3,40 y 3,90, estos son resultados alentadores si se considera la extensión y complejidad del método y el reducido número de tareas para el logro de la independencia cognoscitiva. Ello sugiere continuar trabajando con el método de solución de tareas experimentales cualitativas de biología en otras asignaturas biológicas a fin de consolidar el desarrollo de la habilidad. Sin embargo, estos ya se consideran resultados alentadores en tanto que todas las etapas se encuentran por encima del aprobado.

- La prueba de los efectos intersujetos para el factor fijo estudiantes devolvió el valor de significación de $0,000<0,05$, lo que evidencia diferencias entre los estudiantes. Este resultado es lógico, dado que cada uno tiene su propio ritmo de aprendizaje.

Los resultados para medias individuales de los estudiantes son los siguientes: los 
estudiantes 1 y 10 bajaron a 2,75. Ambos estudiantes abandonaron la universidad posteriormente. El estudiante 8 se mantuvo en una media de 3,00 y el resto de los estudiantes tuvo avances con medias entre 3,38 y 4,73 (tabla 3, tercer ensayo). Los resultados del tercer ensayo se consideran alentadores, razón por la cual se decidió culminar con el proceso de intervención práctica.

\section{Tabla 5}

Prueba de Duncan para las etapas del método de solución de tareas experimentales cualitativas de Biología. Tercer ensayo.

\begin{tabular}{lcc}
\hline \multicolumn{2}{c}{$\begin{array}{c}\text { Variable dependiente: independencia cognoscitiva en la aplicación del método de } \\
\text { solución de tareas experimentales cualitativas de biología }\end{array}$} \\
\hline \multicolumn{1}{c}{ Etapas método } & $\mathrm{N}$ & Grupos homogéneos \\
\cline { 3 - 3 } & 10 & Grupo único \\
\hline Contrastación teórica de los resultados & 10 & 3,40 \\
Proposición de la nueva tarea & 10 & 3,40 \\
Comprensión de la tarea & 10 & 3,60 \\
Control valorativo & 10 & 3,70 \\
Ejecución del plan de la solución & 10 & 3,80 \\
Confección del plan de la solución & 10 & 3,80 \\
Ejecución del experimento y procesamiento de los datos & 10 & 3,90 \\
Diseño del experimento & &, 064 \\
Sig. & & \\
\hline
\end{tabular}

De manera general, los resultados alcanzados en la intervención práctica evidencian que el trabajo con las tareas experimentales cualitativas de biología requiere:

- La orientación con tiempo de antelación de cada tarea, pues producto de la complejidad estructural del método, no da tiempo a realizar la solución completa en una sola clase de dos horas.

- La planificación de consultas y controles individuales, lo que exige un mayor fondo de tiempo para el profesor.

- Continuar con la aplicación del método en el resto de las disciplinas biológicas para lograr su consolidación y sistematización.

Los resultados alcanzados en los ensayos y los análisis cualitativos realizados en las fases intermedias evidenciaron que, desde el punto de vista didáctico, el método de solución propuesto puede emplearse para lograr la independencia cognoscitiva al resolver las tareas experimentales cualitativas de biología. Estos resultados son una modesta prueba de la hipótesis científica enunciada al inicio, aunque se sugiere continuar realizando intervenciones prácticas para evidenciar la validez del método con mayor fortaleza.

\section{Conclusiones}

La estructura del método de solución de tareas experimentales cualitativas de biología se concibió a partir de la analogía —en correspondencia con los criterios de Leyva (2002)—, y está conformada por las etapas: comprensión de la tarea, confección del plan de la solución, 
ejecución del plan de la solución, diseño del experimento, ejecución del experimento y procesamiento de los datos, control valorativo del curso de la solución y de los resultados, contrastación teórica de los resultados y proposición de la nueva tarea. Cada una de estas etapas cuenta con procedimientos mediante los cuales se realiza.

El experimento de modelo probó la validez del método de solución de tareas experimentales cualitativas de biología. Se evidenció su funcionalidad tanto en cantidad, como en variedad de tareas en que es posible aplicarlo, lo cual posibilita el logro de la sistematicidad requerida para el diseño de las asignaturas biológicas.

La intervención práctica evidenció lo siguiente:

- Los estudiantes muestran tendencia a la ejecución, lo que sugiere explicitar cada una de las etapas del método y ofrecer niveles de ayuda.

- Las etapas del método que más tiempo requieren para lograr la independencia cognoscitiva son la contrastación de los resultados y la proposición de la nueva tarea, lo que sugiere un mayor énfasis didáctico para su asimilación.

- La independencia cognoscitiva depende del ritmo de aprendizaje de los estudiantes, lo que exige la atención de las diferencias individuales.

- El logro de la independencia cognoscitiva requiere transitar por tres fases: dominio inicial, dominio formal y consolidación; lo que, en la intervención llevada a cabo, abarcó 21 tareas experimentales, extendidas a más de una asignatura biológica.

- Las tareas deben ser orientadas con antelación y se sugiere complementar la actividad independiente del estudiante con consultas y controles individuales.

\section{Referencias}

Álvarez, J., \& Valls, C. (2019). Utilización de la contextualización mediante el uso de demostraciones experimentales para mejorar la percepción y la actitud hacía la química de los futuros maestros. Enseñanza de las ciencias, 37(3), 73-88. https://doi.org/10.5565/ rev/ensciencias. 2674

Barolli, E., Laburú, C., \& Guridi, V. (2010). Laboratorio didáctico de ciencias: caminos de investigación. Revista Electrónica de Enseñanza de las Ciencias, 9(1), 88-110.

Benoit, C., Castro, R., \& Jaramillo, C. (2019). Aprendizaje y formación valórica en la enseñanza mediante tutorías entre pares. Praxis \& Saber, 10(22), 89-113. https://doi. org/10.19053/22160159.v10.n22.2019.8796

Caamaño, A. (2012). ¿Cómo introducir la indagación en el aula? Revista Alambique: Didáctica de las Ciencias Experimentales, 70, 83-92.

Campistrous, L., \& Rizo, C. (2002). Aprende a resolver problemas aritméticos. Pueblo y Educación.

Carp, D., García, D., \& Chiacchiarini, P. (2012). Trabajos prácticos de laboratorio sin receta de cocina en cursos masivos. Revista Avances en Ciencia e Ingeniería, 3(1), 167-173.

Crujeiras, B., \& Cambeiro, F. (2018). Una experiencia de indagación cooperativa para 
aprender ciencias en educación secundaria participando en las prácticas científicas. Revista Eureka sobre Enseñanza y Divulgación de las Ciencias, 15(1), 1201-1-1201-9. https://doi.org/10.25267/Rev_Eureka_ensen_divulg_cienc.2018.v15.11.1201

Crujeiras, B., \& Jiménez, M. (2015). Desafíos planteados por las actividades abiertas de indagación en el laboratorio: articulación de conocimientos teóricos y prácticos en las prácticas científicas. Enseñanza de las Ciencias, 33(1), 63-84. https://doi.org/10.5565/ rev/ensciencias. 1469

Crujeiras, B., \& Jiménez, M. (2018). Influencia de distintas estrategias de andamiaje para promover la participación del alumnado de secundaria en las prácticas científicas. Enseñanza de las ciencias, 36(2), 23-42. https://doi.org/10.5565/rev/ensciencias.2241

Domenech, J. (2013). Secuencias de apertura experimental y escritura de artículos en el laboratorio: un itinerario de mejora de los trabajos prácticos en el laboratorio. Revista Enseñanza de las Ciencias, 31(3), 249-262.

Espinosa-Ríos, E., González-López, K., \& Hernández-Ramírez, L. (2016). Las prácticas de laboratorio: una estrategia didáctica en la construcción de conocimiento científico escolar. Entramado, 12(1), 266-281. http://dx.doi.org/10.18041/entramado.2016v12n1.23125

Fernández, N. (2013). Los trabajos prácticos de laboratorio por investigación en la enseñanza de la biología. Revista de Educación en Biología, 16(2), 15-30.

Ferrés-Gurt, C. (2017). El reto de plantear preguntas científicas investigables. Revista Eureka sobre Enseñanza y Divulgación de las Ciencias, 14(2), 410-426. http://dx.doi. org/10.25267/Rev_Eureka_ensen_divulg_cienc.2017.v14.i2.09

Galagovsky, L., \& Adúriz-Bravo, A. (2001). Modelos y analogías en la enseñanza de las ciencias naturales. El concepto de modelo didáctico analógico. Enseñanza de las Ciencias, 19(2), 231-242.

García, D. (2019). El método de solución de tareas experimentales cualitativas de Biología como contenido de la enseñanza [Tesis doctoral no publicada]. Universidad Central Marta Abreu de las Villas, Cuba

García, D., Leyva, J., \& Guerra, Y. (2017). Cuadro de diseño del experimento para resolver tareas experimentales de biología. Revista Varela, 17(48), 364-378.

Gil, J. (2017). Rasgos del profesorado asociados al uso de diferentes estrategias metodológicas en las clases de ciencias. Enseñanza de las Ciencias, 35(1), 175-192. http://dx.doi. org/10.5565/rev/ensciencias. 1970

Gómez, C., Solaz, J., \& Sanjosé, V. (2013). Efectos de la similitud superficial y estructural sobre la transferencia a partir de análogos en problemas de alta y baja familiaridad: primeros resultados. Revista Enseñanza de las Ciencias, 31(1), 135-151.

González, C., Martínez, M., Martínez, C., Cuevas, K., \& Muñoz, C. (2009). La educación científica como apoyo a la movilidad social: desafíos en torno al rol del profesor secundario en la implementación de la indagación científica como enfoque pedagógico. Revista Estudios Pedagógicos, 35(1), 63-78. https://doi.org/10.4067/S0718-07052009000100004

González, R., \& Crujeiras, P. (2016). Aprendizaje de las reacciones químicas a través de 
actividades de indagación en el laboratorio sobre cuestiones de la vida cotidiana. Revista Enseñanza de las Ciencias, 34(3), 143-160. http://dx.doi.org/10.5565/rev/ ensciencias.2018

Grilli-Silva, J. (2018). El material natural en la biología escolar. Consideraciones éticas y didáctica sobre las actividades prácticas de laboratorio. Revista Eureka sobre Enseñanza y Divulgación de las Ciencias, 15(1), 1104-1-1104-19. https://doi.org/10.25267/Rev_ Eureka_ensen_divulg_cienc.2018.v15.i1.1104

Guerra, Y. (2008). Modelo didáctico para la implementación de los métodos numéricos en el proceso docente educativo de la física general en la especialidad de profesor de ciencias exactas [Tesis doctoral, Instituto Superior Pedagógico Félix Varela Morales, Cuba]. http://dspace.uclv.edu.cu/handle/123456789/8850

Hernández-Junco, L., Machado-Bravo, E., Martínez-Sardá, E., Andreu-Gómez, N., \& Flint, A. (2018). La práctica de laboratorio en la asignatura Química General y su enfoque investigativo. Revista Cubana Química, 30(2), 314-327.

Hodson, D. (1994). Hacia un enfoque más crítico del trabajo de laboratorio. Revista Enseñanza de las Ciencias, 12(3), 299-313.

Kiruschkin, D., Shapovalenko, S., \& Polosin, V. (1981). Selección de temas de metodología de la enseñanza de la Química. Pueblo y Educación.

Labarrere, A. (1996). Pensamiento. Análisis y autorregulación de la actividad cognoscitiva de los alumnos. Pueblo y Educación.

Landau, L., Sileo, M., \& Lastres, L. (1997). Transformación de un trabajo práctico tradicional. Revista Educación Química, 8(4), 200-204.

Leyva, J. (2002). La estructura del método de solución de tareas experimentales de Física como invariante del contenido [Tesis doctoral, Instituto Superior Pedagógico Félix Varela Morales, Cuba]. http://dspace.uclv.edu.cu/handle/123456789/12561

Leyva, J., \& Guerra, Y. (2005). Las habilidades extendidas. Revista Varela, (10).

Machado, E. (2004). Integración del componente académico con el laboral e investigativo a partir de actividades experimentales [Tesis doctoral no publicada]. Instituto Superior Pedagógico Félix Varela Morales, Cuba

Ministerio de Educación Superior. (2016). Carrera Licenciatura en Educación. Biología. Plan de estudio E. Curso diurno. Curso encuentro. República de Cuba.

Muschietti-Piana, M. del P, Civeira, G., \& Muschietti-Piana, M. (2017). La intervención docente en educación universitaria: una experiencia con prácticas de laboratorio para estudiantes de ciencias ambientales. Revista de Educación en Biología, 20(2), 28-40.

Peres, F., \& Marques, C. (2013). Problematización de las actividades experimentales en la formación y la práctica docente de los formadores de profesores de química. Revista Enseñanza de las Ciencias, 31(3), 67-86.

Rivero, H. (2003). Un modelo para el tratamiento didáctico integral de las tareas teóricas de Física y su solución [Tesis doctoral no publicada]. Instituto Superior Pedagógico Félix 
Varela Morales, Cuba.

Siso, Z., Briceño, J., Álvarez, C., \& Arana, J. (2009). Las prácticas de laboratorio en la formación del profesorado de química. Un primer acercamiento. Revista Electrónica Diálogos Educativos, 9(18),139-161.

Via, A., \& Izquierdo, M. (2016). Aprendizaje por competencias (I). Identificación de los perfiles de las competencias adquiridas. Enseñanza de las Ciencias, 34(3), 73-90. http:// dx.doi.org/10.5565/rev/ensciencias.1693 